\title{
Recent Advances in Lipid-Based Formulation Technology
}

\author{
Christopher J. H. Porter • Hywel D. Williams • Natalie L. Trevaskis
}

Received: 10 October 2013 / Accepted: 10 October 2013 / Published online: 26 October 2013

(C) Springer Science+Business Media New York 2013

\section{INTRODUCTION}

Lipid-based formulations encompass a wide variety of delivery modes and technologies, ranging from immediate release to sustained release formulations, from liquids to semi-solids, from crystalline suspensions to lipid solutions, from systems containing small-molecule active pharmaceutical ingredients (APIs) to those containing more complex peptide-based molecules, and finally from those intended for oral administrations through to topicals and parenterals. The flexibility of lipid formulation technologies provides the potential to modify pharmaceutical properties such as stability and ease of manufacture, and pharmacokinetic properties such as enhanced absorption and bioavailability, sustained exposure and targeted delivery. With the exception of liposomal technologies (that represent a class of delivery system in their own right and have not been considered here), much of the interest in lipid-based formulations has focused on their use to enhance the oral bioavailability of poorly water-soluble drugs. Drugs with low water solubility continue to present a formidable challenge to effective drug development (1) and commonly show poor, variable, and fooddependent absorption and bioavailability after oral administration. Whilst great strides have been made in lead optimization strategies to design-out the physicochemical properties that lead to low water solubility, in many cases, structural changes that increase solubility also result in unacceptable reductions in potency. Indeed, the majority of reports describing changes in

C. J. H. Porter $(\bowtie) \cdot H$. D. Williams $\cdot$ N. L. Trevaskis

Drug Delivery Disposition and Dynamics

Monash Institute of Pharmaceutical Sciences

Monash University (Parkville campus)

Melbourne, Victoria, Australia

e-mail: chris.porter@monash.edu

H. D. Williams

e-mail: hywel.williams@capsugel.com

N. L. Trevaskis

e-mail: natalie.trevaskis@monash.edu

Present Address:

H. D. Williams

Capsugel R\&D Strasbourg, France the patterns of physicochemical properties for new drug discovery candidates suggest that trends towards low water solubility are increasing rather than decreasing $(2,3)$. Formulation strategies that facilitate the 'rescue' of compounds with intrinsically low water solubility, and that provide for robust and reproducible exposure after oral administration, therefore remain an important weapon in the drug development armory.

Several strategies that enable effective delivery of poorly water-soluble compounds are apparent (1) and, in general, may be stratified as technologies that modify solute-solute interactions in the solid state, that enhance solute-solvent interactions in solution, that promote dissolution via changes in surface area, or combinations of the three approaches. Formulation technologies to enhance solubility and dissolution rate include salt formulation, modification of crystal form (amorphous, cocrystals, polymorphs), particle size reduction/nanomilling, alteration of solution/solubilisation conditions (cosolvents, surfactants, cyclodextrins etc.), and approaches such as solid dispersions and lipidbased formulations that may, in practice, achieve a number of these goals. For example, many solid dispersion and lipid-based formulations contain drug in a non-crystalline (amorphous/molecularly dispersed) form, circumventing the challenge to solubility provided by the crystal lattice, and also provide for ongoing solubilisation or stabilization of supersaturation via the inclusion of surfactants, lipids and/or polymers.

The current theme issue focuses on the potential utility of lipid-based formulations as a means of enhancing the oral bioavailability of poorly water-soluble drugs. In addition, several manuscripts provide insight into the broader application of lipid-based drug delivery technologies, for example, to facilitate parenteral or site-specific delivery of lipophilic compounds. This is not to suggest any inherent superiority of lipid-based formulation technologies over others, and the appropriate choice of an enabling technology is clearly drug specific. However, for poorly water-soluble lipophilic drugs, lipid-based formulations provide a tried and tested delivery solution to overcome poor and variable oral bioavailability and are increasingly being utilized as a vehicle to achieve modulated or targeted delivery.

Nonetheless, whilst lipid-based formulations have been used to good effect in a range of oral and parenteral drug products (4), several challenges to their broader utility remain. These 
include an improved understanding of the mechanisms by which lipid-based formulations enhance oral bioavailability, and in particular the role of supersaturation in promoting drug absorption; the development of in vitro and/or in silico analytical tools that are better able to predict in vivo utility; formulation approaches that allow conversion of lipid-based formulations to solid dose forms thereby facilitating tablet formation; improved control of drug stability in lipid-based formulations; and enhanced understanding of the ability of lipid-based formulations to facilitate site-specific delivery (such as delivery to the lymph or spatial and temporal control of delivery kinetics). These topics are specifically addressed in the current theme issue.

\section{MECHANISTIC EVALUATION OF THE UTILITY OF LIPID-BASED FORMULATIONS}

Several studies in the current issue have addressed the mechanisms that underpin the utility of lipid-based formulations. Elgart et al. assess two self-emulsifying formulations of amiodarone and talinolol in rats and show significant increases in bioavailability in both cases after administration of the lipid formulation. Subsequent studies suggest that the lipid formulation leads to increases in gastrointestinal (GI) solubilisation and absorptive permeability, and in the case of talinolol in particular, possible effects on P-glycoprotein efflux. In a separate approach, Yeap et al. explore the potential for lipid-based formulations of cinnarizine to promote drug absorption via the induction of supersaturation at the absorptive membrane. Using a series of in situ intestinal perfusion and bioavailability studies, the authors show that lipid absorption from intestinal mixed micelles at the absorptive membrane is likely to reduce micellar solubilisation capacity, promote supersaturation, and drive increases in drug absorption. The data therefore suggest that formulations containing absorbable lipids may provide inherent advantage in the promotion of supersaturation and, as a result, in enhancing the absorption of poorly water-soluble drug cargoes. The role of supersaturation in drug absorption is an area of increasing interest across the discipline, and the integration of lipid-based formulations into in vivo lipid processing pathways appears to provide multiple means by which supersaturation can be generated. These include formulation digestion, interaction with biliary amphiphiles, and stimulation of lipid absorption. These concepts and the broader implications of lipid-based formulations as a means to stimulate supersaturation are explored and expanded in a mini-review by Williams and co-authors.

\section{IN VITRO ASSESSMENT TOOLS FOR LIPID-BASED FORMULATIONS}

Lipid-based formulations are unlike many typical oral formulations in that they show two-way in vivo interactivity. Thus, lipids and surfactants in the formulations can initiate significant physiological change via the stimulation of biliary and pancreatic secretions, changes to GI transit, and alterations to cellular permeability. In parallel, normal GI physiology can markedly affect formulation functionality via initiation of lipid digestion and bile-mediated solubilisation. These multifaceted interactions dictate that the development of simple in vitro tests that are reflective of in vivo performance is complex and our understanding in this area continues to evolve. In the current issue, Williams et al. report on the latest data from the Lipid Formulation Classification System (LFCS) Consortium, a group of academic and industrial scientists that was established to develop more robust in vitro tools to assess lipid-based formulation performance, with a particular focus on lipid digestion. The latest data from the Consortium report the performance of 48 different formulation/drug/drug loading combinations during digestion and show that the threshold for precipitation of a poorly water-soluble drug (PWSD) appears to be remarkably consistent. Thus, drug precipitation under digesting conditions occurred only where the supersaturation ratio was greater than 3 , regardless of the drug or formulation employed, providing a potential indicator of maximum drug load in lipid-based formulations. Fernandez and colleagues also report on the use of in vitro digestion tests to evaluate the enzymatic processing of Labrasol ${ }^{\circledR}$, an excipient in common use in lipid formulations. The authors describe the digestion of Labrasol ${ }^{\circledR}$ by both duodenal and gastric lipases and reveal that the activity of the different GI enzymes toward this excipient is heavily dependent on dilution and differences in the colloidal structures that are formed on dilution.

As it becomes clearer that relatively subtle changes in colloidal structure may be capable of dictating significant differences in enzyme activity and therefore drug solubilisation, tools to more robustly evaluate evolving structures during intestinal processing of lipid-based formulations become more important. To this end, Phan et al. describe methods to allow detailed characterization of the structures formed during the digestion of common formulation components using synchrotron small angle X-ray scattering (sSAXS) and cryogenic transmission electron microscopy (cryo-TEM). The former allows much more rapid acquisition of SAXS data and therefore real-time monitoring of phase changes.

Larsen et al. also employed in vitro digestion tests in an attempt to better understand the patterns of drug absorption from a series of self-emulsifying formulations of cinnarizine. In these studies the relative ratios of drug to formulation were systematically altered and effects on in vitro digestion and in viwo drug exposure assessed. Cinnarizine oral bioavailability in dogs was surprisingly similar, regardless of the drug loading, in spite of in vitro data suggesting that performance would deteriorate at the higher loadings. The data reinforce that there remains 
much to learn in how best to interpret in vitro digestion data when attempting to forecast in vivo exposure, and that these relationships are likely drug specific. Notwithstanding these recent data for cinnarizine, a number of publications have previously described studies where precipitation during in vitro digestion correlates well with reductions in in vivo exposure (5-8). Consideration of the kinetics of drug precipitation therefore remains an important experimental endpoint. Real-time assessment of precipitation during the dynamic process of lipid digestion, however, is challenging, and most studies currently examine precipitation off-line. To address this limitation, Stilhart et al. explore the use of a Raman probe to allow in situ detection of crystallization from digesting lipid-based formulations containing fenofibrate. The combination of real-time Raman spectroscopy and mathematical modeling provides insight into the kinetics of digestion-triggered supersaturation and drug crystallization and allows more effective timeresolution of precipitation events.

Buyukozturk et al. took a slightly different approach and developed a systems-based model to predict the dissolution rate of a model "drug" (in this case, an EPR spin probe) in the presence of digesting food-associated lipids or when suspended in a self-emulsifying lipid formulation. The model, based on inputs including drug particle size, solubility, lipid droplet size, and digestion rate, highlights the importance of lipid digestion in initiating drug solubilization and, in combination with pharmacokinetic models of drug disposition, may ultimately provide a means of better predicting food effects and the in vivo utility of lipid-based formulations.

A significant body of work has examined the impact of digesting lipids on drug solubilisation. Far fewer have sought to probe the impact of drug solubilisation in lipid colloidal phases on intestinal permeability. In general, solubilisation in the colloidal structures formed via dispersion and digestion of lipid-based formulations reduces thermodynamic activity and might therefore be expected to reduce flux and bioavailability at a fixed drug dose. In the current issue, Markopoulos et al. examine this issue directly and using PAMPA assays examine the flux of a range of poorly water soluble drugs across model membranes from both model intestinal fluids and aspirated human intestinal fluid. Whilst permeability was found to decrease in the presence of solubilizing species, total drug flux did not alter significantly, since the solubilizing species simultaneously allowed the maintenance of higher total drug concentrations.

In a separate but related in vitro examination Modi and Anderson used model liposome systems to explore drug-lipid bilayer partitioning and used these studies to develop models of the $\mathrm{pH}$ dependence of membrane partitioning for ionisable compounds. The data suggest that the contributions of both ionized and unionized species must be accounted for, and that bilayer free surface energy may be an important determinant of the lipid partition coefficient. This contrasts with the common practice of using partitioning in bulk lipids (e.g. octanol) to provide an indication of in vivo membrane partitioning and has implications for the design of partitioning measurements with improved relevance for passive membrane transport and biodistribution.

\section{MODIFIED LIPID-BASED DELIVERY SYSTEMS}

The potential advantages of lipid-based formulations have been well described and are further exemplified in the current theme issue. Liquid filled capsules, however, require access to specialized equipment, be that a means to seal hard gelatin capsules or access to soft gelatin encapsulation technologies. While liquid-filled capsules are increasingly employed, there are some efforts to explore the feasibility of converting lipidbased liquid formulations to solid formulations, for potential tablet manufacture or capsule powder fill. In the current issue, Serajuddin and coworkers describe two studies that explore the solidification of lipid-based formulations via adsorption onto high surface area silicates. In the first study, the authors show that of many silicates explored, only Neusilin® US2 could yield tablets with acceptable tensile strength when mixed in a 1:1 weight ratio with the lipid formulation. The same group subsequently explored Neusilin ${ }^{\circledR}$ US2-based formulations in more detail and showed that effective drug release was possible in most cases, but that under some conditions "gel formation" on contact with water prevented drug release by blocking water ingress into the porous architecture of the silicate. The basic principles of solidification of lipidbased formulations are more broadly addressed by a review article from the Prestidge group.

In a separate approach, Tokle et al. explore the potential utility of modifying the colloid interface of lipid droplets using nanolamination. The lamination process involved sequential electrostatic deposition of cationic lactoferrin and anionic $\beta$ lactoglobulin to the surface of a corn oil emulsion. The authors show that modified emulsions could be readily generated with potentially improved stability and that the coating had little impact on digestibility in an in vitro digestion model, suggesting rapid removal of the protein coatings in vivo. Interestingly, the transfer of a model poorly soluble cargo ( $\beta$ carotene) from the oil droplets to the aqueous solubilised phase in the in vitro digestion test was very low. The authors suggested that this was due to complexation with cationic lactoferrin (or a lactoferrin digestion product) that may or may not occur in vivo, reinforcing the importance of considering and/or monitoring for drug solubilisation alongside lipid digestion.

Mengesha et al. also explored the utility of a modified lipid formulation, but in this case one designed for parenteral administration rather than oral administration. More specifically, the systems examined were designed to allow for site-specific drug release via activation using an external 
magnetic field. Lipid matrices containing blends of glyceryl monooleate and glyceryl monostearate were first evaluated in order to identify mixtures with gel-phase transition temperatures slightly above physiological (in this case $42^{\circ}$ ). Matrices were then loaded with iron oxide nanoparticles that were modified with oleic acid to enhance miscibility with the lipid matrix. The iron oxide nanoparticles rendered the system suitable for remote activation since the application of an alternating magnetic field induced sufficient nanoparticle heating to stimulate the phase change in the lipid, thereby activating pulsatile drug release from the matrix.

\section{COMPUTATIONAL APPROACHES TO LIPID FORMULATION DESIGN}

In silico approaches have the potential to dramatically enhance the speed of drug development and the same is true for the development of lipid-based formulations. Of the many areas where computational approaches may assist in formulation development, two have been addressed in detail in the current theme issue. Persson et al. present a detailed assessment of the solubility of 30 diverse drugs in long and medium chain glyceride lipids, polysorbate 80 and PEG, and utilize this data to produce a principle component analysis of the variance in lipid solubility. The data suggest strong correlation between medium and long chain lipid solubilities and between PEG and polysorbate 80 solubility, raising the possibility that solubility in one could be used to predict the other. Solubilities in medium and long chain triglycerides were also predicted de novo using calculated molecular descriptors, even in the absence of melting point information. In a different in silico approach, Warren et al. used molecular dynamics to simulate the patterns of formulation interaction with water in the presence and absence of drug. Formulation micro-structure was evident from the simulations at all water concentrations, and as the water content increased, the microstructure evolved from a continuous phase containing isolated water molecules, to a reverse-micellar solution and finally to a system containing lamellar lipids with large pools of free water. Molecular dynamics also provided some insight into the preferential positioning of drugs at the interface or within the lipid-rich or water-rich areas of the formulation.

\section{DRUG STABILITY IN LIPID-BASED FORMULATIONS}

Relatively little has been published on the chemical stability of drugs in lipid-based formulations, even though practitioners acknowledge that this can be a complex area. In the absence of a significant literature base to review, Stella provides a timely overview and commentary, based on many years of experience, of the potential hurdles and pitfalls of drug stability in lipid based formulations. From this commentary, and the examples Stella provides, it is clear that greater appreciation of the complexities of drug stability in lipid-based formulations is required and that research programs that seek to evaluate these complexities and to develop strategies to circumvent these problems are long overdue.

\section{LIPID-BASED FORMULATIONS FOR INTESTINAL LYMPHATIC TRANSPORT}

In addition to supporting drug solubilisation and in some cases enhancing intestinal permeability, lipid-based formulations may also promote the intestinal lymphatic transport of highly lipophilic drug candidates. In this issue Trevaskis et al. report on the development of a mouse model to assess lymphatic transport. This was developed to allow the conduct of lymphatic transport studies in the species in which most activity studies are performed (i.e., mice) and to allow more ready access to transgenic animals. The authors also used the mouse data to compare to previous lymphatic transport data in rats and dogs and showed that lymphatic lipid transport scaled linearly on a $\mathrm{mg} / \mathrm{kg}$ basis across species. In contrast, lymphatic drug transport decreased from dog to rat to mouse. This was suggested to reflect both a decrease in drug bioavailability in the lower order species and a reduction in intrinsic lymphatic transport efficiency. Future studies will address the mechanistic basis behind these observations. In a more fundamental study of the mechanisms that underpin lymphatic lipid transport, Dixon et al. report that contrary to most literature descriptions, active (ATP dependent) transport systems may exist to promote lymphatic lipid access raising the interesting possibility that affinity for that transport process may dictate, at least in part, patterns of lymphatic drug transport.

\section{SUMMARY}

Lipid-based formulations may be used to enhance the delivery of a range of drug molecules via oral and parenteral administration. The current theme issue brings together submissions from a range of contributors and has focused largely on lipid based formulations to enhance the oral absorption of poorly water soluble drugs, since this continues to be a major impediment to drug development. It is also apparent, however, that lipids can provide advantage beyond solubility enhancement, including improvements in permeability, stability and lymphatic transport, and can provide flexible platforms for the development of stimuli-sensitive parenteral depots. These aspects are also addressed. 


\section{REFERENCES}

1. Williams HD, Trevaskis NL, Charman SA, Shanker RM, Charman WN, Pouton CW, et al. Strategies to address low drug solubility in discovery and development. Pharmacol Rev. 2013;65:315-499.

2. Leeson PD, Springthorpe B. The influence of drug-like concepts on decision-making in medicinal chemistry. Nat Rev Drug Discov. 2007;6:881-90.

3. Leeson PD, St-Gallay SA. The influence of the 'organizational factor' on compound quality in drug discovery. Nat Rev Drug Discov. 2011;10:749-65.

4. Strickley RG. Solubilizing excipients in oral and injectable formulations. Pharm Res. 2004;21:201-30.

5. Cuiné JF, Charman WN, Pouton CW, Edwards GA, Porter CJH. Increasing the proportional content of surfactant (Cremophor EL) relative to lipid in self-emulsifying lipid-based formulations of danazol reduces oral bioavailability in beagle dogs. Pharm Res. 2007;24: 748-57.

6. Dahan A, Hoffman A. Use of a dynamic in vitro lipolysis model to rationalize oral formulation development for poor water soluble drugs: correlation with in vivo data and the relationship to intra-enterocyte processes in rats. Pharm Res. 2006;23:2165-74.

7. Cuiné JF, McEvoy CL, Charman WN, Pouton CW, Edwards GA, Benameur $\mathrm{H}$, et al. Evaluation of the impact of surfactant digestion on the bioavailability of danazol after oral administration of lipidic self-emulsifying formulations to dogs. J Pharm Sci. 2008;97:995-1012.

8. Fatouros DG, Nielsen FS, Douroumis D, Hadjileontiadis LJ, Mullertz A. In vitro-in vivo correlations of self-emulsifying drug delivery systems combining the dynamic lipolysis model and neuro-fuzzy networks. Eur J Pharm Biopharm. 2008;69:887-98.

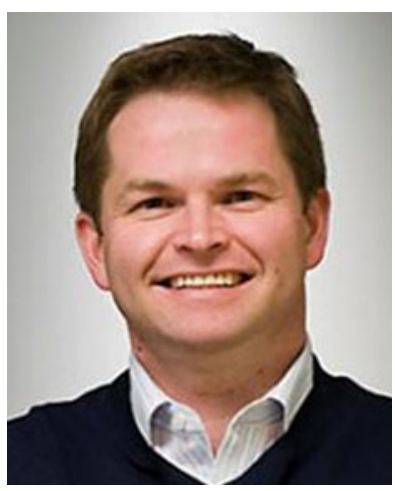

Chris Porter is Professor of Pharmaceutics at the Monash Institute of Pharmaceutical Sciences and Associate Dean (Research) of the Faculty of Pharmacy and Pharmaceutical Sciences at Monash University, Melbourne. He completed his undergraduate and graduate studies at the University of Nottingham before moving to Australia and Monash in 1992. Subsequently, Chris' research programs have focused on the impact of formulation on drug absorption, distribution and elimination profiles and on developing the models and techniques to probe these interactions. A major interest has been the issues and problems surrounding the absorption of poorly water soluble, lipophilic drugs and the use of lipid based delivery systems to enhance oral bioavailability and stimulate lymphatic transport. More recently, his interests have expanded into mechanisms of intracellular drug transport and the potential utility of dendrimers as drug delivery systems. Chris has published more than 150 peer reviewed papers in these areas and his programs have attracted $\sim \$ 10 \mathrm{~m}$ in funding from government and commercial organisations. Chris is a current member of the Editorial Boards of Pharmaceutical Research, the Journal of Pharmaceutical Sciences and the Journal of Pharmacy and Pharmacology.

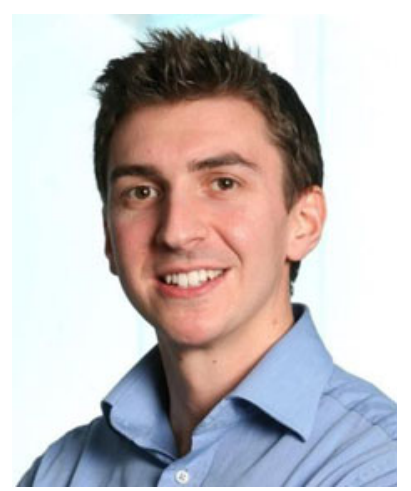

Dr. Hywel D. Williams is a Senior Scientist working on innovative lipid-based technologies in the Pharmaceutical Sciences Department of Capsugel R\&D in Strasbourg, France. He received his Masters in Pharmacy degree at the Welsh School of Pharmacy in Cardiff, Wales, UK and then his $\mathrm{PhD}$ in Pharmaceutical Sciences at the University of Nottingham, UK. After completing his $\mathrm{PhD}$, Dr. Williams spent 3 years as a research fellow at the Monash Institute of Pharmaceutical Sciences, Melbourne, Australia working principally within the LFCS Consortium on establishing standardized tests for lipid formulations and identifying the key factors that impact their performance. Dr. Williams sits on the Capsugel Scientific Advisory Committee, is a member of the steering committee for the Lipid-Based Drug Delivery Systems Focus Group of the AAPS and remains an adjunct faculty member at Monash University. He has published 11 first/second author research papers in controlled/lipid drug delivery, in addition to review articles and numerous conference oral and poster presentations.

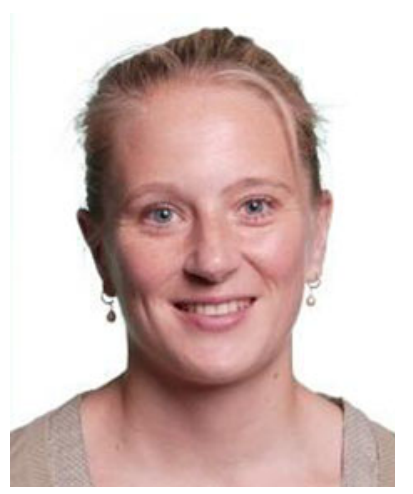

Dr. Natalie L Trevaskis is a Research fellow in Drug Delivery, Disposition and Dynamics at the Monash Institute of Pharmaceutical Sciences, Melbourne, Australia. Her research focuses on understanding the mechanisms by which lipid-based formulations promote the intestinal absorption and lymphatic transport of lipophilic compounds and more recently, the use of lipids to promote drug delivery to immune and metabolic targets. She obtained a Bachelor of Pharmacy with Honours and $\mathrm{PhD}$ in

Pharmaceutical Sciences from Monash University for which she received the Gold Medal and Mollie Holman Doctoral Medal, respectively. Her $\mathrm{PhD}$ examined the biological mechanisms which control lipid and drug transport via the intestinal lymphatics and included a period of study in the Metabolic Diseases Institute, University of Cincinnati. In projects with Pfizer, Novartis and Astra Zeneca she has subsequently studied the mechanisms by which co-administration with lipids promotes the absorption and lymphatic transport of lipophilic drug candidates, and influences their PK and/or PD. In projects supported by the National Health and Medical Research Council of Australia she has examined the mechanisms by which lipid formulations promote lipophilic drug absorption, and the potential to improve the treatment of immune and metabolic disease by targeting drug delivery to the lymphatics. Dr. Trevaskis has published 22 research papers and has contributed to over 30 conference presentations. She is a scientific advisor for the Journal of Pharmaceutical Sciences and lectures in pharmacokinetics at Monash University. 